\title{
Planar graphene Josephson coupling via van der Waals superconducting contacts
}

\section{DOI:}

10.1016/j.cap.2018.06.016

\section{Document Version}

Accepted author manuscript

Link to publication record in Manchester Research Explorer

\section{Citation for published version (APA):}

Lee, J., Kim, M., Watanabe, K., Taniguchi, T., Lee, G. H., \& Lee, H. J. (2019). Planar graphene Josephson coupling via van der Waals superconducting contacts. Current Applied Physics, 19(3), 251-255.

https://doi.org/10.1016/j.cap.2018.06.016

\section{Published in:}

Current Applied Physics

\section{Citing this paper}

Please note that where the full-text provided on Manchester Research Explorer is the Author Accepted Manuscript or Proof version this may differ from the final Published version. If citing, it is advised that you check and use the publisher's definitive version.

\section{General rights}

Copyright and moral rights for the publications made accessible in the Research Explorer are retained by the authors and/or other copyright owners and it is a condition of accessing publications that users recognise and abide by the legal requirements associated with these rights.

\section{Takedown policy}

If you believe that this document breaches copyright please refer to the University of Manchester's Takedown Procedures [http://man.ac.uk/04Y6Bo] or contact uml.scholarlycommunications@manchester.ac.uk providing relevant details, so we can investigate your claim.

\section{OPEN ACCESS}




\title{
Planar graphene Josephson coupling via van der Waals superconducting contacts
}

Jongyun Lee ${ }^{\mathrm{a}}$, Minsoo Kim ${ }^{\mathrm{a},}$, Kenji Watanabe ${ }^{\mathrm{b}}$, Takashi Taniguchi ${ }^{\mathrm{b}}$, Gil-Ho Lee ${ }^{\mathrm{a}}$, and HuJong Lee ${ }^{\mathrm{a}}$

${ }^{a}$ Department of physics, Pohang University of Science and Technology, Pohang 37673, Korea

${ }^{\mathrm{b}}$ National Institute for Materials Science, Tsukuba 305-0044, Japan

KEYWORDS: graphene, proximity Josephson junction, Van der Waals materials, Van der Waals stacking, dry transfer

\begin{abstract}
We report on the fabrication and transport characteristics of van der Waals (vdW)-contacted planar Josephson junctions. In a device, two pieces of cleaved $2 \mathrm{H}-\mathrm{NbSe} \mathrm{e}_{2}$ superconducting flakes and a monolayer graphene sheet serve as the superconducting electrodes and the normal conducting spacer, respectively. A stack of $\mathrm{NbSe}_{2}-$ graphene-hexagonal boron nitride $(\mathrm{hBN})$ heterostructure with clean and flat interfaces was prepared by a dry transfer technique. The outermost hBN layer protected the $\mathrm{NbSe}_{2}-$ graphene- $\mathrm{NbSe}_{2}$ Josephson junction from chemical contamination during the fabrication processes. The Josephson coupling was confirmed by a periodic modulation of the junction critical current $I_{\mathrm{c}}$ in a perpendicular magnetic field. The temperature dependence of $I_{c}$ showed long and diffusive Josephson coupling characteristics. The temperature dependence of the superconducting gap, obtained from the multiple Andreev reflection features, followed the Bardeen-Cooper-Schrieffer (BCS) prediction.
\end{abstract}

* Present address: School of Physics \& Astronomy, University of Manchester, Manchester M13 9PL, United Kingdom 
† E-mail: hjlee@ postech.ac.kr 


\section{Introduction}

Since the realization of graphene layers, a variety of two-dimensional (2D) materials have been produced by the mechanical exfoliation technique [1], including metals [2, 3], insulators [4, 5], semiconductors [6], topological insulators [7, 8], superconductors [1, 9-11], etc. Fundamental properties of various cleavable 2D materials including graphene have been investigated extensively to date, together with exploring their electronic applications $[12,13]$. Recently developed dry transfer technique has allowed to stack such cleaved 2D materials into van der Waals (vdW) heterostructures with atomically flat and clean interfaces $[14,15]$, which often reveal novel physical properties $[16,17]$. Proximity Josephson coupling is a good example: when a normal conductor $(\mathrm{N})$ is sandwiched between two closely spaced superconductors (S), a supercurrent can flow through the $\mathrm{N}$ layer without dissipation, forming an SNS proximity Josephson junction.

Such a SNS junction requires electrically transparent interfaces between the $\mathrm{S}$ and $\mathrm{N}$ layers. However, conventional ways of depositing superconducting materials with electronbeam/thermal evaporation or plasma sputtering, with highly energetic evaporants, may seriously damage the normal conducting insert and the $\mathrm{S} / \mathrm{N}$ interfaces. Such deterioration can be minimized by dry transferring superconducting materials onto the normal conducting insert as demonstrated in previous studies $[18,19]$. Recently realization of vertical Josephson junctions has been demonstrated by vdW staking of two dry transferred superconducting layers onto each other. Here, the angle mismatch of the two stacked $2 \mathrm{H}-\mathrm{NbSe}_{2}$ flakes leads to formation of a weak-link with tunneling-like junction behavior [20]. On the other hand, vertically stacked $\mathrm{NbSe}_{2}-$ graphene- $\mathrm{NbSe}_{2}$ vdW junctions has shown strong proximity Josephson coupling [21]. $\mathrm{NbSe}_{2}$ is one of cleavable superconducting materials, maintaining superconductivity down to a few atomic layers [9-11, 22]. Other form of vertical Josephson junctions were fabricated by electron beam deposition of aluminum superconducting electrodes on both sides of graphene [23]. Although the short and ballistic strong proximity Josephson coupling was demonstrated in the vertical junctions, characteristics of the graphene layer were not gate tunable due to screening of the gating field by the superconducting electrodes.

In this study, we report on the fabrication and the measurements of vdW-contacted planar proximity Josephson junctions by employing $\mathrm{NbSe}_{2}$ flakes as superconducting electrodes and a graphene layer as a normal-conducting weak-link spacer. Dry transfer technique [14] 
enabled to form superconducting interfaces which were sufficiently transparent as to exhibit Josephson coupling. The planar junction geometry allowed electrostatic tuning of Josephson coupling by modulating carrier density of the graphene weak link. To form an $\mathrm{NbSe}_{2}$-graphene- $\mathrm{NbSe}_{2}$ planar Josephson junction, we dry-transferred [14] graphene-hexagonal-boron-nitride (hBN) bilayer onto two pieces of $\mathrm{NbSe}_{2}$ flakes which were closely pre-arranged. The outermost hBN layer protected the Josephson junction from the chemicals and ambient moistures during the fabrication process. Thus-prepared junction exhibited the Josephson current up to temperature of $T \sim 1 \mathrm{~K}$. We investigated the nature of Josephson coupling by examining the temperature and magnetic-field dependences of the

junction critical current $I_{\mathrm{c}}$. The temperature dependence of $I_{\mathrm{c}}$ showed that the junction was in a long diffusive regime [24]. Gate dependence of the Josephson coupling and Fraunhofer interference under magnetic field were also investigated. This study demonstrates the potentials of vdW heterostructured superconducting hybrid quantum devices [25-27].

\section{Device fabrication and basic characteristics}

Figures 1(a-f) illustrates device fabrication processes. The hBN flakes were mechanically exfoliated and identified on $\mathrm{SiO}_{2}$ substrate coated with a thin polypropylene carbonate (PPC) layer. After peeling off a PPC layer from a $\mathrm{SiO}_{2}$ substrate, it was transferred onto a Gel-pak layer. Using dry transfer method [14], a graphene layer was picked up by an hBN layer. Consequently, a graphene-hBN bilayer stack was transferred onto two separate pieces of $\mathrm{NbSe}_{2}$ flakes, which were closely pre-arranged with parallel edges as shown in Fig. 1(a). The $\mathrm{NbSe}_{2}$ flakes were cover-protected by a graphene-hBN bilayer within an hour of intermission after the exfoliation of $\mathrm{NbSe}_{2}$, which was to minimize the surface degradation of $\mathrm{NbSe}_{2}$ [20]. The Gel-pak stamp released the entire vdW structure at $100{ }^{\circ} \mathrm{C}$ by melting PPC layer [Fig. 1(b)], followed by vacuum annealing to remove PPC residues [Fig. 1(c)]. Scanning electron microscope (SEM) image of Fig. 1(g) was taken at this stage of fabrication. The hBN layer was partially etched using $\mathrm{CF}_{4}$ plasma to make electrode contact holes [Fig. 1(d)]. Ti/Au $(5 / 100 \mathrm{~nm}$ in thickness) bilayer contact electrodes was deposited in sequence right after the in-situ etching of degraded $\mathrm{NbSe}_{2}$ surface by Ar ion milling without breaking the vacuum [Fig. 1(e)]. During fabrication processes, air could have been trapped in the cavity between two $\mathrm{NbSe}_{2}$ flakes and graphene. A small hole of $500 \mathrm{~nm}$ in diameter (black dotted circle in Fig. 1(h)) was made by using $\mathrm{CF}_{4}$ plasma etching and Ar ion milling [Fig. 1(f)]. It was to 
pump out the air trapped in the cavity and thus to minimize the hysteresis with respect to backgating. Figure 1(h) shows an optical image of the device A together with the measurement configuration. Graphene is denoted by a white dashed line. All of the data in this report, except for the Fig. 3(b), were taken from the device A. The key in this fabrication process was that the graphene and $\mathrm{NbSe}_{2}$ were not exposed to chemicals during the fabrication processes. The graphene layer was suspended from the substrate by the thickness of the $\mathrm{NbSe}_{2}$ electrodes, which helped maintain its cleanness. Figure 1(i) shows gate-voltage $\left(V_{\mathrm{G}}\right)$ dependence of the normal-state junction resistance $\left(R_{\mathrm{N}}\right) . R_{\mathrm{N}}$ is asymmetric with respect to the charge neutral point (CNP). The work function of $\mathrm{NbSe}_{2}, \sim 5.9 \mathrm{eV}$ [28], is larger than that of graphene, $\sim 4.5 \mathrm{eV}[29,30]$, so that the graphene layer in contact with $\mathrm{NbSe}_{2}$ electrodes was hole-doped. A slight increase of $R_{\mathrm{N}}$ in the electron-doped regime $\left(V_{\mathrm{G}}>0\right)$ is attributed to the formation of less transparent $\mathrm{p}-\mathrm{n}-\mathrm{p}$ junction by the work function mismatch between graphene and $\mathrm{NbSe}_{2} . V_{\mathrm{G}}$ dependence of the junction conductance in out-of-plane magnetic fields is plotted in Fig. 1(j). In high magnetic fields, the junction shows clear

quantized plateaus of $G=v e^{2} / h$ with filling factor of $v=4\left(n+\frac{1}{2}\right)=2,6,10$ (red horizontal lines; $n=$ the Landau-level index). These quantized half-integer quantum-Hall conductance plateaus confirm that the graphene layer used in the measurements was a monolayer $[2,3]$. The plateaus are clearer at negative $V_{G}$ than positive side (not shown) due to the hole-doping of the graphene at the contacts with $\mathrm{NbSe}_{2}$.

\section{Results and discussion}

Current-voltage (I-V) characteristic curves of Fig. 2(a) were taken at base temperature of 15 $\mathrm{mK}$. The $I-V$ characteristics show sharp transitions from a superconducting to resistive state at $I_{\mathrm{C}}$. In addition, $V_{\mathrm{G}}$ changes both $I_{\mathrm{c}}$ and $R_{\mathrm{N}}$. There is a negligible hysteresis in $I_{\mathrm{c}}$ and retrapping current $\left(I_{\mathrm{r}}\right)$, indicating that the junction is in an overdamped state [31]. Figure 2(b) shows the differential resistance as a function of $V_{\mathrm{G}}$ and the bias current. The upper and lower boundaries between the dark-blue (zero $\mathrm{d} V / \mathrm{d} I$ ) and light-blue regions (finite $\mathrm{d} V / \mathrm{d} I$ ) represent $I_{\mathrm{c}}$ and $I_{\mathrm{r}}$, respectively. The overlapped red curve represents $V_{\mathrm{G}}$ dependence of the normal-state conductance. $I_{c}$ shows an asymmetric gate dependence across the CNP, similar to the feature appeared in normal-state junction conductance. Although the graphene layer between the $\mathrm{NbSe}_{2}$ flakes was electron-doped by the positive $V_{\mathrm{G}}$, the graphene region 
in contact with the $\mathrm{NbSe}_{2}$ remained to be hole-doped, which led to the formation of a p-n-p junction with weakened Josephson coupling.

The temperature dependence of $I_{\mathrm{c}}$ for various $V_{\mathrm{G}}$ obtained from the device A is plotted in Fig. 3(a). Data are fitted with the long-junction behavior, the temperature dependence of which is written as,

$$
e I_{\mathrm{c}} R_{\mathrm{N}}=\alpha a E_{\mathrm{Th}}\left[1-b \exp \left(-\frac{a E_{\mathrm{Th}}}{3.2 k_{\mathrm{B}} T}\right)\right]
$$

where $E_{\mathrm{Th}}=\hbar D / L^{2}$ is the Thouless energy, $\hbar$ is the Planck constant divided by $2 \pi$, and $a$ and $b$ are phenomenological fitting parameters [24]. Here, $D=v_{\mathrm{F}} l_{\mathrm{mfp}} / 2$ is the carrier diffusion constant in the graphene layer, where $v_{\mathrm{F}}$ is the Fermi velocity and $l_{\mathrm{mfp}}$ is the mean free path. $\alpha$ is introduced to reflect reduction of the junction current due to low contact transparency, Fermi-velocity mismatch, and Fermi-level pinning. Using the data of Fig. 1(i), $E_{\mathrm{Th}}$ and $l_{\mathrm{mfp}}$ are estimated to be $50 \mu \mathrm{eV}$ and $30 \mathrm{~nm}$, respectively, for $V_{\mathrm{G}}=$ $-50 \mathrm{~V}$. As $l_{\mathrm{mfp}} \sim 30 \mathrm{~nm}$ is smaller than the channel length of the device A, $L=450 \mathrm{~nm}$, the junction is in a diffusive limit. In a long junction limit of $E_{\mathrm{Th}} / \Delta_{\mathrm{NbSe}_{2}} \rightarrow 0$, the parameters are predicted to be $a=10.8$ and $b=1.30$ [24]. Here, $\Delta_{\mathrm{NbSe}_{2}}$ is the superconducting gap of $\mathrm{NbSe}_{2}$. The best fitting with the data gives $a=1.7 \sim 2.7, b=1.4 \sim 1.7$ and $\alpha=$ 0.10 0.14 with $E_{\mathrm{Th}} / \Delta_{\mathrm{NbSe}_{2}}=0.057$. The device A falls into the intermediate regime between the short $\left(E_{\mathrm{Th}} / \Delta_{\mathrm{NbSe}_{2}}>1\right)$ and the long $\left(E_{\mathrm{Th}} / \Delta_{\mathrm{NbSe}_{2}}<0.01\right)$ junction limits. The fitting parameter $a$ was indeed smaller than 10.8 as expected in the intermediate regime between the long and short junction limits $[24,32]$. The temperature dependence of $I_{\mathrm{c}}$ for the device B of $L=150 \mathrm{~nm}$ is shown in Fig. 3(b). $I_{\mathrm{c}}-T$ curves shows a convex upward behavior at low temperatures, which is reminiscent of a short-junction behavior [21, 23, 33]. However, the $I_{\mathrm{c}}$ data at higher temperatures are required to claim the short Josephson coupling characteristics.

The periodic modulation of $I_{\mathrm{c}}$ in varying magnetic fields, so-called the Fraunhofer diffraction pattern, shown in Fig. 4 is a firm evidence of the Josephson junction [31]. Colorcoded differential resistance maps are plotted as a function of magnetic field and bias current for various $V_{\mathrm{G}}$ [Fig. 4]. Boundaries between the zero-resistance and resistive regions represent $I_{\mathrm{c}}$ and $I_{\mathrm{r}}$. $I_{\mathrm{c}}$ shows local minima when integer multiples of magnetic flux quanta penetrate the junction area. $I_{\mathrm{c}}$ clearly oscillates in magnetic-field periods of $B_{0}=$ 
$\Phi_{0} /[(L+2 \lambda) W] \sim 2.0 \mathrm{G}$, where $\Phi_{0}=h / 2 e$ is the magnetic-flux quantum, $W$ is the junction width, and $\lambda$ is the London penetration depth of the superconducting $\mathrm{NbSe}_{2}$ flakes $[31,34]$. The period $B_{0}$ is similar at different $V_{\mathrm{G}}$ as indicated by red vertical dashed lines. When current distribution is uniform along the width of the junction, the magnetic field dependence of $I_{\mathrm{c}}$ follows the relation of $I_{\mathrm{c}}(B)=I_{0}\left|\sin \left(\pi \Phi / \Phi_{0}\right) /\left(\pi \Phi / \Phi_{0}\right)\right|$, where $\Phi=B[(L+2 \lambda) W]$ is the magnetic flux penetrating the junction area $[31,34]$. The solid lines are the fitting curves to the experimental results. The magnetic field period of the oscillation $B_{0} \sim 2.01 \mathrm{G}$ yields $L+2 \lambda \sim 4.1 \mu \mathrm{m}$. While the London penetration depth $\lambda$ of the bulk $\mathrm{NbSe}_{2}$ is known to be $130 \mathrm{~nm}$ [35], it should be replaced by an expression, $\lambda_{2 \mathrm{D}} \sim 2 \lambda^{2} / d$, for a 2D superconductor [36], which turns out to be $1.5 \mu \mathrm{m}$ for the $\mathrm{NbSe}_{2}$ electrodes with the thickness of $d=23 \mathrm{~nm}$ as used in this study. The corresponding field penetration range becomes $L+2 \lambda_{2 \mathrm{D}} \sim 3.5 \mu \mathrm{m}$, which is in reasonable agreement with the value determined from the data, $4.1 \mu \mathrm{m}$.

Lastly, we discuss the bias-voltage dependence of the differential resistance shown in Fig. 5(a), exhibiting the multiple Andreev reflection (MAR) at low temperatures, which is a distinctive feature of proximity-type Josephson junctions [37, 38]. Multiple Andreev reflection exhibits differential resistance dips indicated by a red dotted line in Fig. 5(a) at the voltage of $V_{\mathrm{MAR}}=2 \Delta_{\mathrm{NbSe}_{2}} /$ ne [37, 38], with the integer number corresponding to $n=2$. As temperature increases, $V_{\text {MAR }}$ decreases due to the reduction of superconducting gap energy and vanishes above critical temperature of $\mathrm{NbSe}_{2}$ as shown in Fig. 5(b). The device A consists of two $\mathrm{NbSe}_{2}$ flakes of slightly different critical temperatures, $T_{\mathrm{c}, \mathrm{u}} \sim 5.3 \mathrm{~K}$ for the upper flake and $T_{\mathrm{c}, \mathrm{I}} \sim 5.6 \mathrm{~K}$ for the lower one as indicated in the inset of Fig. 2(a). To analyze the temperature dependence of $V_{\mathrm{MAR}}$ for a Josephson junction consisting of two superconductors of different $T_{\mathrm{c}}$, we introduce the combined superconducting gap of $\Delta_{0}(T)=2 \Delta_{\mathrm{u}} \Delta_{\mathrm{l}} /\left[\Delta_{\mathrm{u}}+\Delta_{\mathrm{l}}\right]$, where $\Delta_{\mathrm{u}}$ and $\Delta_{\mathrm{l}}$ are the superconducting gaps of upper and lower $\mathrm{NbSe}_{2}$ flakes, respectively [23, 39]. Here, we assumed the temperature dependence of $\Delta_{\mathrm{u}}$ and $\Delta_{\mathrm{l}}$ following the Bardeen-Cooper-Schrieffer (BCS) theory and the zerotemperature superconducting gap $\Delta_{\mathrm{u} / \mathrm{l}}(T=0)=\gamma k_{\mathrm{B}} T_{\mathrm{c}, \mathrm{u} / \mathrm{l}}$ with a proportionality factor $\gamma$ [40]. The solid pink line in Fig. 5(b) represents the best fit with $V_{\text {MAR }}$ for the value of $\gamma=1.72$, which agrees well with the BCS theory of 1.76 [40]. This gives $\Delta_{0}(T=0) \sim 0.81$ meV. 


\section{Conclusions}

In conclusion, we successfully fabricated vdW-stacked planar $\mathrm{NbSe}_{2}-$ graphene- $\mathrm{NbSe}_{2}$ Josephson junctions by using the dry transfer technique [14]. The outermost hBN layer protected the entire device structure from the chemical contamination during the fabrication processes. This made it possible to fabricate Josephson junctions based on the vdW superconductor, $\mathrm{NbSe}_{2}$, even being exposed to air. The temperature dependence of $I_{\mathrm{c}}$ shows a typical long-diffusive Josephson-junction behavior [24]. The Fraunhofer interference in outof-plane magnetic fields confirms Josephson coupling with a uniform current distribution along the width of the junction. Multiple Andreev reflection signatures further confirm proximity-type of the Josephson coupling. This study reports on the first realization of vdWcontacted planar Josephson junctions by dry transfer technique, which would pave a road to a potential application of vdW-stacked heterostructures for superconducting hybrid quantum devices.

\section{Acknowledgments}

This work was supported by the National Research Foundation of Korea (NRF) through the SRC Center for Topological Matter, POSTECH, Korea (Grant No. 2011-0030046 for H.-J.L.), the SRC Center for Quantum Coherence in Condensed Matter, KAIST, Korea (Grant No. 2016R1A5A1008184 for G.-H.L.), and the Elemental Strategy Initiative conducted by the MEXT and JSPS KAKENHI, Japan (Grant Number JP15K21722 for K.W. and T.T.). 


\section{Figures and captions}

(a)

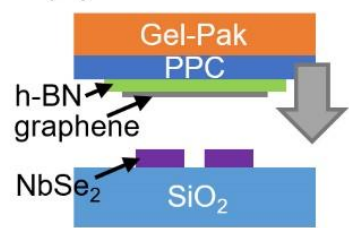

(d)

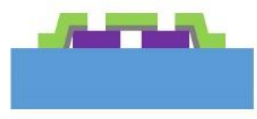

(g)

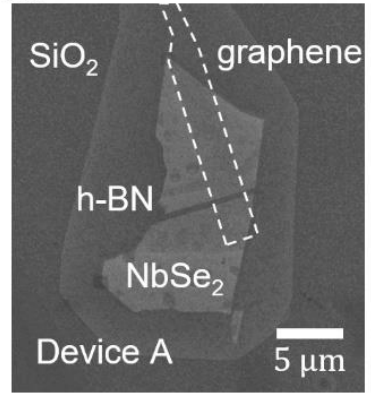

(i)

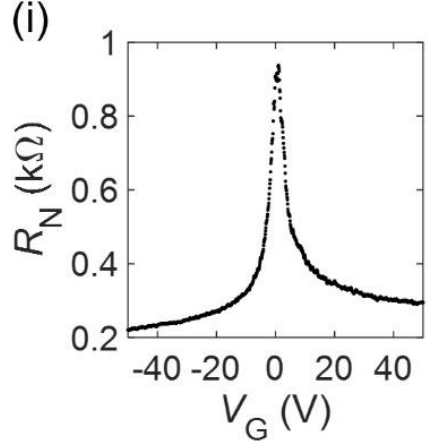

(e) (c)
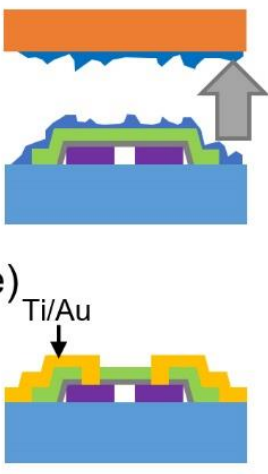

(f)

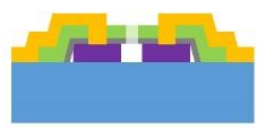

(h)

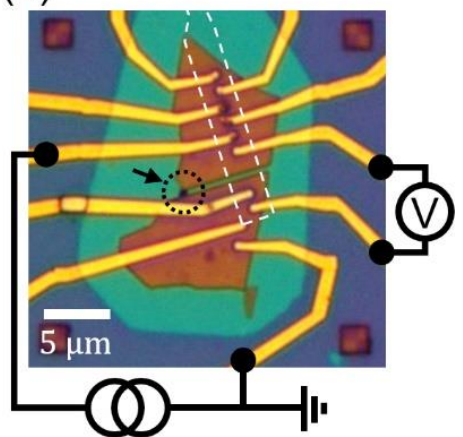

(j)

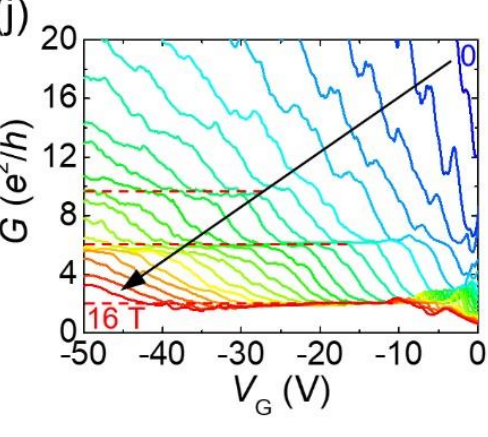

Figure 1. Device fabrication process and normal-state properties of graphene. (a-f) Schematics of the fabrication processes. (g) SEM image of a multilayered heterostructure of Fig. 1(c). The thickness of $\mathrm{NbSe}_{2}(23 \mathrm{~nm})$ and $\mathrm{hBN}(30 \mathrm{~nm})$ flakes were measured by atomic force microscope. (h) Optical microscope image of the fabricated device and measurement configurations. After loading a device in a sealed can, the trench cavity was pumped out through a hole, denoted in the black dotted circle, to remove any contaminant trapped inside the trench cavity. (i) Gate-voltage dependence of the normal-state junction resistance obtained from the device A at $T=15 \mathrm{~K}$. (j) The gate-voltage dependence of the conductance in out-of-plane magnetic fields, ranging from 0 to $16 \mathrm{~T}$. 
(a)

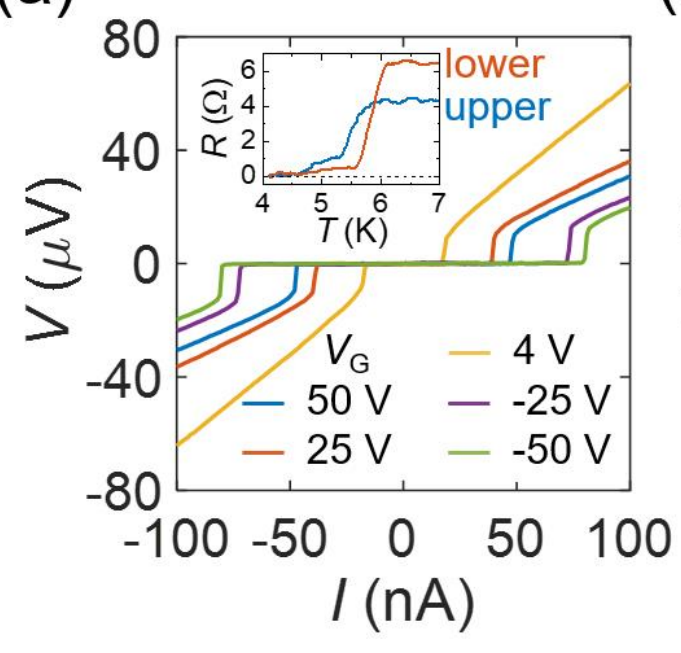

(b)

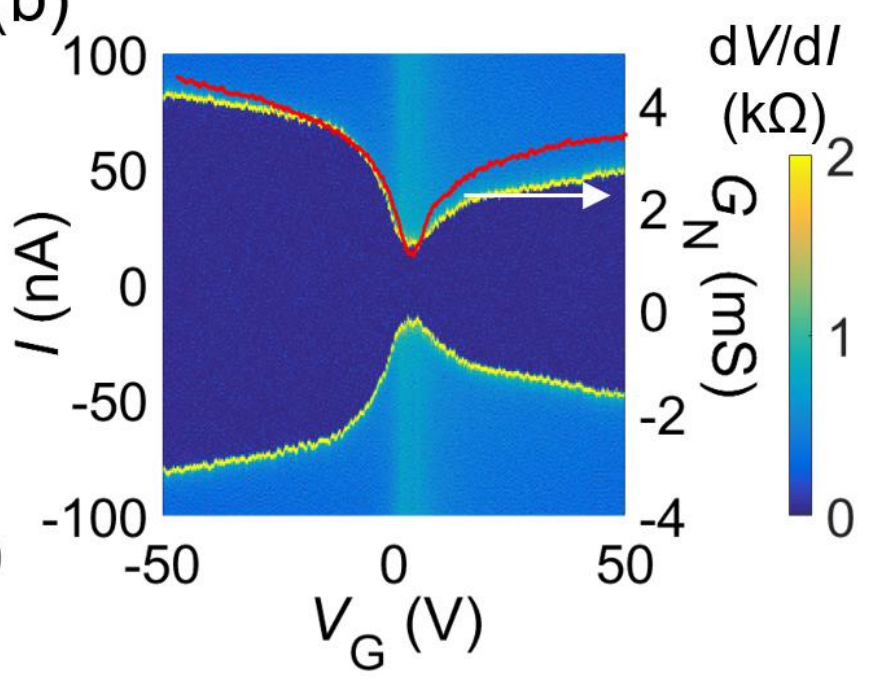

Figure 2. Basic Josephson junction properties. (a) Current-voltage (I-V) characteristic curves of the device A for different gate voltages, $V_{\mathrm{G}}$. Inset: resistance vs temperature $(R-T)$ curves of the upper and lower $\mathrm{NbSe}_{2}$ flakes, showing the critical temperature of the upper $\mathrm{NbSe}_{2}$ flake $T_{\mathrm{c}, \mathrm{u}} \sim 5.3 \mathrm{~K}$ and the lower one $T_{\mathrm{c}, \mathrm{l}} \sim 5.6 \mathrm{~K}$. (b) Differential-resistance map of the device $\mathrm{A}$ as a function of the gate voltage $V_{\mathrm{G}}$ and the bias current $I$. Red curve shows the gatevoltage dependence of the normal-state junction conductance. 

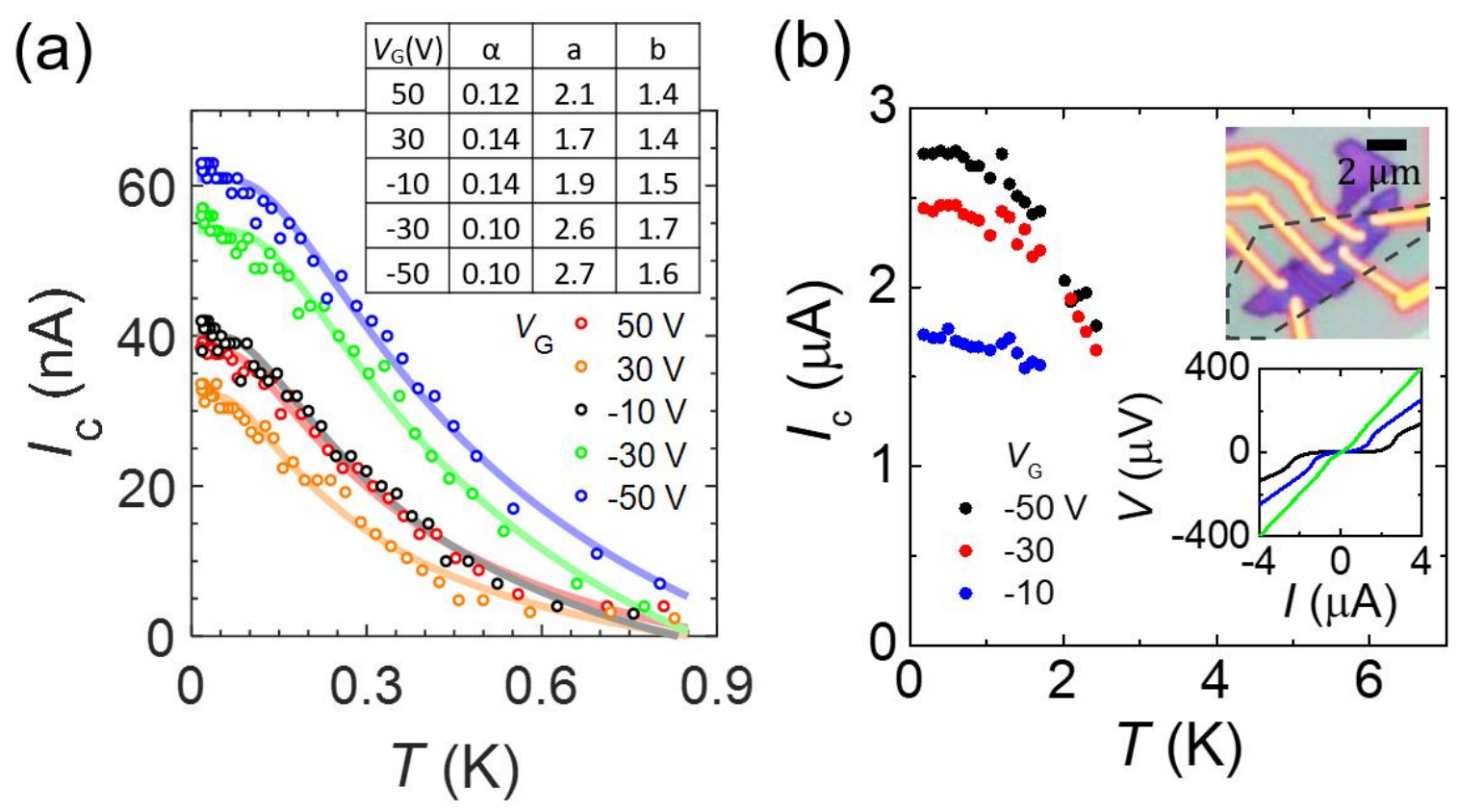

Figure 3. Temperature dependence of the junction critical current $I_{c}$ obtained for (a) the device $\mathrm{A}$ and (b) the device $\mathrm{B}$ at various gate voltages $V_{\mathrm{G}}$. (a) Solid curves are the best fits with the long-diffusive junction behavior of $I_{\mathrm{c}}$ with corresponding parameters listed in the table. (b) Upper inset: Optical microscope image of the fabricated device B. Grey dashed line indicates the graphene boundary. Lower inset: Current-voltage (I-V) characteristic curves of the device $\mathrm{B}$ for different gate voltages, $V_{\mathrm{G}}$. The critical current $I_{\mathrm{c}}$ in the main panel is determined by the bias currents giving rise to maxima of the differential resistance $\mathrm{d} V / \mathrm{d} I$ of the $I-V$ curves. 


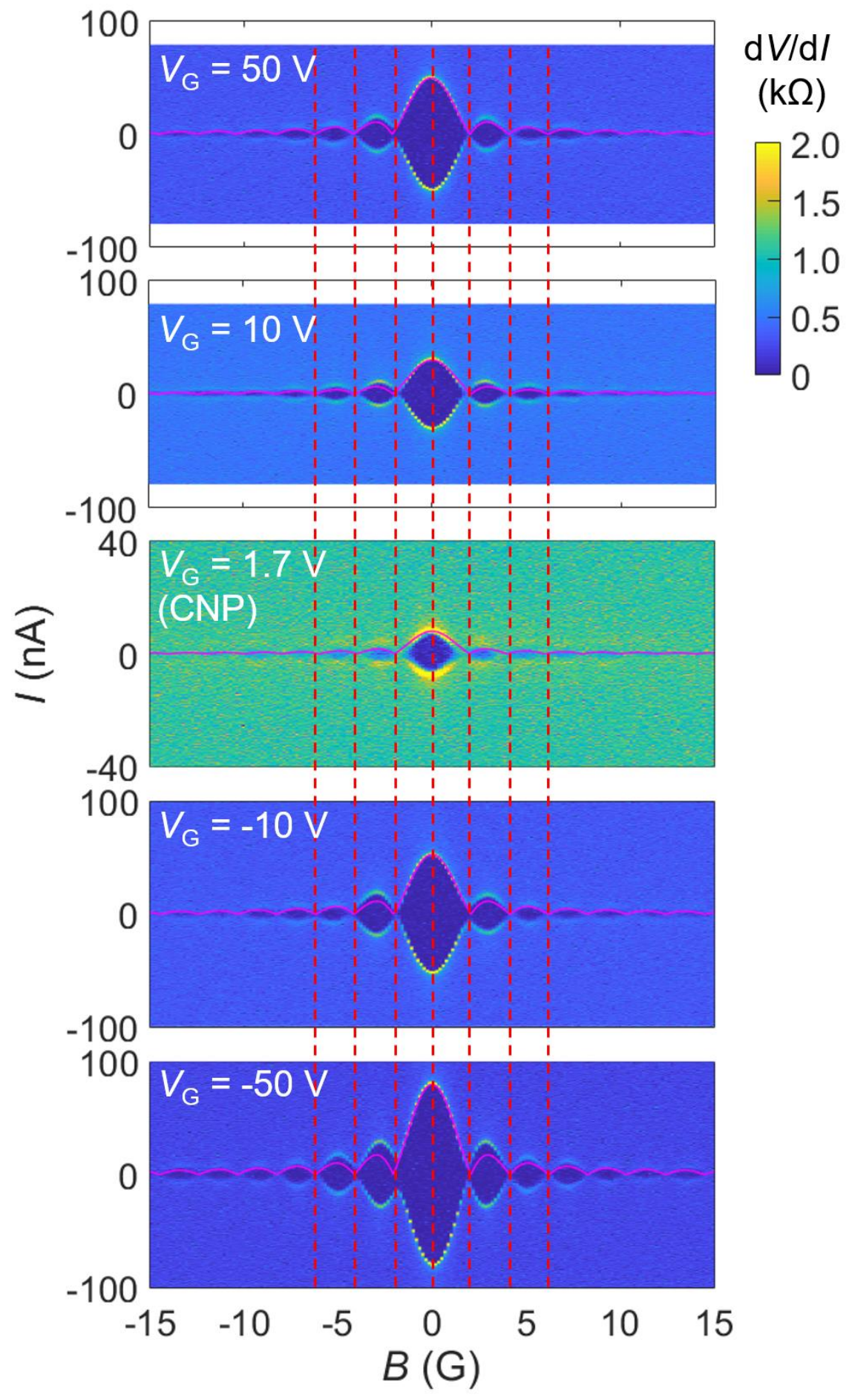

Figure 4. Magnetic field dependence of the critical current; Fraunhofer interference pattern. Differential resistance map of the device A as a function of magnetic field and bias current for various gate voltages, $V_{\mathrm{G}}$. Red vertical lines indicate the period of $I_{\mathrm{c}}$ oscillation. Magenta solid lines represent theoretical fitting of the Fraunhofer pattern with a uniform current distribution along the junction width. 
(a)

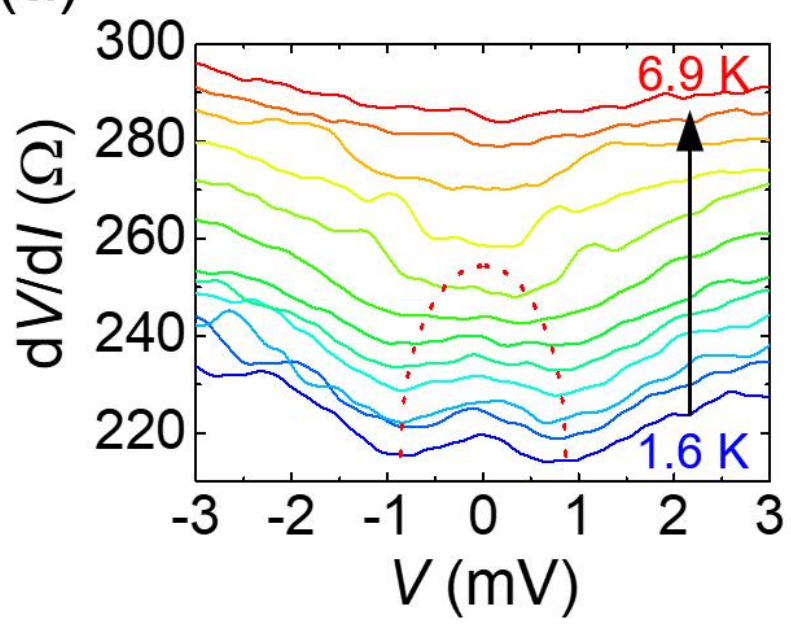

(b)

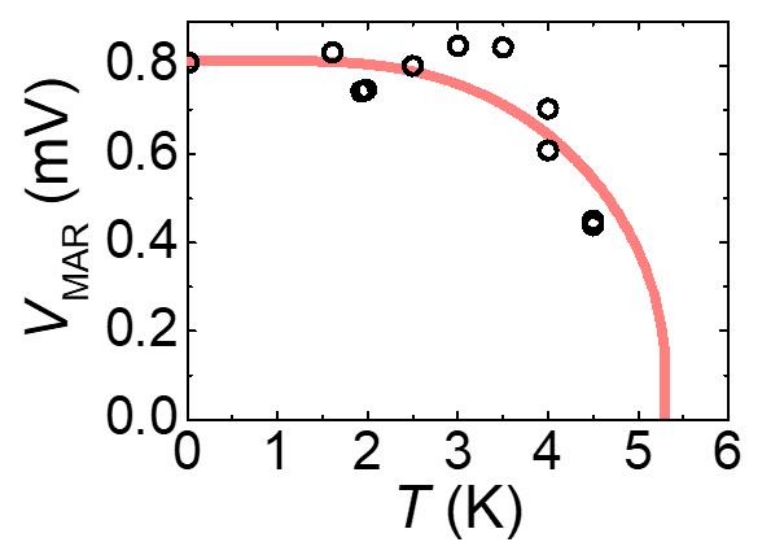

Figure 5. Temperature dependence of the multiple Andreev reflection (MAR) signature. (a) Bias-voltage dependence of the differential resistance obtained from the device A, measured at temperatures ranging from 1.6 to $6.9 \mathrm{~K}$. The red dotted line is a guide to the eye, corresponding to the local minima of the differential resistance. (b) Temperature dependence of the differential resistance minima (symbol) and the Bardeen-Cooper-Schrieffer (BCS) fit (solid line). 


\section{References}

[1] K. S. Novoselov, D. Jiang, F. Schedin, T. J. Booth, V. V. Khotkevich, S. V. Morozov, A. K. Geim, Two-dimensional atomic crystals, Proc. Natl. Acad. Sci. U.S.A., 102 (2005) 10451-10453.

[2] K. S. Novoselov, A. K. Geim, S. V. Morozov, D. Jiang, M. I. Katsnelson, I. V. Grigorieva, S. V. Dubonos, A. A. Firsov, Two-dimensional gas of massless Dirac fermions in graphene, Nature, 438 (2005) 197-200.

[3] Y. Zhang, Y.-W. Tan, H. L. Stormer, P. Kim, Experimental observation of the quantum Hall effect and Berry's phase in graphene, Nature, 438 (2005) 201-204.

[4] Y. Kubota, K. Watanabe, O. Tsuda, T. Taniguchi, Deep ultraviolet light-emitting hexagonal boron nitride synthesized at atmospheric pressure, Science, 317 (2007) 932934.

[5] L. A. Ponomarenko, A. K. Geim, A. A. Zhukov, R. Jalil, S. V. Morozov, K. S. Novoselov, I. V. Grigorieva, E. H. Hill, V. V. Cheianov, V. I. Fal'ko, K. Watanabe, T. Taniguchi, R. V. Gorbachev, Tunable metal-insulator transition in double-layer graphene heterostructures, Nat. Phys., 7 (2011) 958-961.

[6] K. F. Mak, C. Lee, J. Hone, J. Shan, T. F. Heinz, Atomically thin $\mathrm{MoS}_{2}$ : A new direct-gap semiconductor, Phys. Rev. Lett., 105 (2010) 136805.

[7] D. Hsieh, D. Qian, L. Wray, Y. Xia, Y. S. Hor, R. J. Cava, M. Z. Hasan, A topological Dirac insulator in a quantum spin Hall phase, Nature, 452 (2008) 970-974.

[8] Y. Xia, D. Qian, D. Hsieh, L. Wray, A. Pal, H. Lin, A. Bansil, D. Grauer, Y. S. Hor, R. J. Cava, M. Z. Hasan, Observation of a large-gap topological-insulator class with a single Dirac cone on the surface, Nat. Phys., 5 (2009) 398-402.

[9] N. E. Staley, J. Wu, P. Eklund, Y. Liu, L. Li, Z. Xu, Electric field effect on superconductivity in atomically thin flakes of $\mathrm{NbSe}_{2}$, Phys. Rev. B, 80 (2009) 184505.

[10] X. Xi, Z. Wang, W. Zhao, J.-H. Park, K.T. Law, H. Berger, L. Forro, J. Shan, K.F. Mak, Ising pairing in superconducting $\mathrm{NbSe}_{2}$ atomic layers, Nat. Phys., 12 (2016) 139-143.

[11] A. W. Tsen, B. Hunt, Y. D. Kim, Z. J. Yuan, S. Jia, R. J. Cava, J. Hone, P. Kim, C. R. Dean, A. N. Pasupathy, Nature of the quantum metal in a two-dimensional crystalline superconductor, Nat. Phys., 12 (2016) 208-212.

[12] M. Xu, T. Liang, M. Shi, H. Chen, Graphene-like two-dimensional materials, Chem. Rev., 113 (2013) 3766-3798.

[13] S. Z. Butler, S. M. Hollen, L. Cao, Y. Cui, J. A. Gupta, H. R. Gutiérrez, T. F. Heinz, S. S. 
Hong, J. Huang, A. F. Ismach, E. Johnston-Halperin, M. Kuno, V. V. Plashnitsa, R. D. Robinson, R. S. Ruoff, S. Salahuddin, J. Shan, L. Shi, M. G. Spencer, M. Terrones, W. Windl, J. E. Goldberger, Progress, challenges, and opportunities in two-dimensional materials beyond graphene, ACS Nano, 7 (2013) 2898-2926.

[14] L. Wang, I. Meric, P. Y. Huang, Q. Gao, Y. Gao, H. Tran, T. Taniguchi, K. Watanabe, L. M. Campos, D. A. Muller, J. Guo, P. Kim, J. Hone, K. L. Shepard, C. R. Dean, Onedimensional electrical contact to a two-dimensional material, Science, 342 (2013) 614617.

[15] S. J. Haigh, A. Gholinia, R. Jalil, S. Romani, L. Britnell, D. C. Elias, K. S. Novoselov, L. A. Ponomarenko, A. K. Geim, R. Gorbachev, Cross-sectional imaging of individual layers and buried interfaces of graphene-based heterostructures and superlattices, Nat. Mater., 11 (2012) 764-767.

[16] A. K. Geim, I. V. Grigorieva, Van der Waals heterostructures, Nature, 499 (2013) 419425.

[17] K. S. Novoselov, A. Mishchenko, A. Carvalho, A. H. Castro Neto, 2D materials and van der Waals heterostructures, Science, 353 (2016) aac9439.

[18] D. K. Efetov, L. Wang, C. Handschin, K. B. Efetov, J. Shuang, R. Cava, T. Taniguchi, K. Watanabe, J. Hone, C. R. Dean, P. Kim, Specular interband Andreev reflections at van der Waals interfaces between graphene and $\mathrm{NbSe}_{2}$, Nat. Phys., 12 (2016) 328-332.

[19] M. R. Sahu, P. Raychaudhuri, A. Das, Andreev reflection near the Dirac point at the graphene-NbSe 2 junction, Phys. Rev. B, 94 (2016) 235451.

[20] N. Yabuki, R. Moriya, M. Arai, Y. Sata, S. Morikawa, S. Masubuchi, T. Machida, Supercurrent in van der Waals Josephson junction, Nat. Commun., 7 (2016) 10616.

[21] M. Kim, G.-H. Park, J. Lee, J. H. Lee, J. Park, H. Lee, G.-H. Lee, H.-J. Lee, Strong proximity Josephson coupling in vertically stacked $\mathrm{NbSe}_{2}-$ graphene- $\mathrm{NbSe}_{2}$ van der Waals junctions, Nano Lett., 17 (2017) 6125-6130.

[22] R. F. Frindt, Superconductivity in ultrathin $\mathrm{NbSe}_{2}$ layers, Phys. Rev. Lett., 28 (1972) 299-301.

[23] G.-H. Lee, S. Kim, S.-H. Jhi, H.-J. Lee, Ultimately short ballistic vertical graphene Josephson junctions, Nat. Commun., 6 (2015) 6181.

[24] P. Dubos, H. Courtois, B. Pannetier, F. K. Wilhelm, A. D. Zaikin, G. Schön, Josephson critical current in a long mesoscopic S-N-S junction, Phys. Rev. B, 63 (2001) 064502.

[25] A. Zazunov, V. S. Shumeiko, E. N. Bratus', J. Lantz, G. Wendin, Andreev level qubit, 
Phys. Rev. Lett., 90 (2003) 087003.

[26] L. Bretheau, Ç. Ö. Girit, H. Pothier, D. Esteve, C. Urbina, Exciting Andreev pairs in a superconducting atomic contact, Nature, 499 (2013) 312-315.

[27] V. S. Pribiag, A. J. A. Beukman, F. Qu, M. C. Cassidy, C. Charpentier, W. Wegscheider, L. P. Kouwenhoven, Edge-mode superconductivity in a two-dimensional topological insulator, Nat. Nanotechnol., 10 (2015) 593-597.

[28] S. Toshihiro, S. O. Fumio, A. P. Bruce, Work function and photothreshold of layered metal dichalcogenides, Jpn. J. Appl. Phys., 33 (1994) 2696-2698.

[29] K. T. Chan, J. B. Neaton, M. L. Cohen, First-principles study of metal adatom adsorption on graphene, Phys. Rev. B, 77 (2008) 235430.

[30] G. Giovannetti, P. A. Khomyakov, G. Brocks, V. M. Karpan, J. van den Brink, P. J. Kelly, Doping graphene with metal contacts, Phys. Rev. Lett., 101 (2008) 026803.

[31] M. Tinkham, Introduction to superconductivity, second ed., Dover Publications, New York, 2004.

[32] D. Jeong, J.-H. Choi, G.-H. Lee, S. Jo, Y.-J. Doh, H.-J. Lee, Observation of supercurrent in PbIn-graphene-PbIn Josephson junction, Phys. Rev. B, 83 (2011) 094503.

[33] J. Park, J. H. Lee, G.-H. Lee, Y. Takane, K.-I. Imura, T. Taniguchi, K. Watanabe, H.-J. Lee, Short ballistic Josephson coupling in planar graphene junctions with inhomogeneous carrier doping, Phys. Rev. Lett., 120 (2018) 077701.

[34] A. Barone, G. Paterno, Physics and Applications of the Josephson Effect, Wiley, New York, 1982.

[35] J. E. Sonier, R. F. Kiefl, J. H. Brewer, J. Chakhalian, S. R. Dunsiger, W. A. MacFarlane, R. I. Miller, A. Wong, G. M. Luke, J. W. Brill, Muon-spin rotation measurements of the magnetic field dependence of the vortex-core radius and magnetic penetration depth in $\mathrm{NbSe}_{2}$, Phys. Rev. Lett., 79 (1997) 1742-1745.

[36] J. Pearl, Current distribution in superconducting films carrying quantized fluxoids, Appl. Phys. Lett., 5 (1964) 65-66.

[37] T. M. Klapwijk, G. E. Blonder, M. Tinkham, Explanation of subharmonic energy gap structure in superconducting contacts, Physica B+C, 109-110 (1982) 1657-1664.

[38] M. Octavio, M. Tinkham, G. E. Blonder, T. M. Klapwijk, Subharmonic energy-gap structure in superconducting constrictions, Phys. Rev. B, 27 (1983) 6739-6746.

[39] K. Böttcher, T. Kopp, Multichannel dc Josephson effect in ballistic point contacts, Phys. Rev. B, 55 (1997) 11670-11687. 
[40] J. Bardeen, L. N. Cooper, J. R. Schrieffer, Theory of superconductivity, Phys. Rev., 108 (1957) 1175-1204. 